\title{
MRI Grading versus Histology: Predicting Survival of World Health Organization Grade II-IV Astrocytomas
}

\author{
A. Lasocki, A. Tsui, M.A. Tacey, K.J. Drummond, K.M. Field, and F. Gaillard
}

\begin{abstract}
BACKGROUND AND PURPOSE: Histologic grading of intracranial astrocytomas is affected by sampling error and substantial inter- and intraobserver variability. We proposed that incorporating MR imaging into grading will predict patient survival more accurately than histopathology alone.
\end{abstract}

\begin{abstract}
MATERIALS AND METHODS: Patients with a new diagnosis of World Health Organization grades II-IV astrocytoma or mixed oligoastrocytoma diagnosed between September 2007 and December 2010 were identified. Two hundred forty-five patients met the inclusion criteria. Preoperative MRIs were independently reviewed by 2 readers blinded to the histologic grade, and an MR imaging grade was given. The MR imaging and histopathologic grades were compared with patient survival.

RESULTS: Patients with grade II or III astrocytomas on histology but evidence of necrosis on MR imaging (consistent with a grade IV tumor) had significantly worse survival than patients with the same histology but no evidence of necrosis on MR imaging $(P=.002$ for grade II histology and $P=.029$ for grade III). Their survival was not significantly different from that in patients with grade IV tumors on histology $(P=.164$ and $P=.385$, respectively); this outcome suggests that all or most are likely to have truly been grade IV tumors. MR imaging evidence of necrosis was less frequent in grade II and III oligoastrocytomas, preventing adequate subgroup analysis.
\end{abstract}

CONCLUSIONS: MR imaging can improve grading of intracranial astrocytomas by identifying patients suspected of being undergraded by histology, with high interobserver agreement. This finding has the potential to optimize patient management, for example, by encouraging more aggressive treatment earlier in the patient's course.

ABBREVIATIONS: ECOG = Eastern Cooperative Oncology Group; $\mathrm{HR}=$ hazard ratio; $I D H I=$ isocitrate dehydrogenase-1

M anagement of intracranial astrocytic tumors is heavily influenced by the histologic grade obtained at the time of surgical biopsy or resection. World Health Organization grade IV astrocytomas (glioblastoma) are the most common, and most glioblastomas are considered to arise de novo (primary glioblastomas). ${ }^{1,2}$ Grade II and III astrocytomas (diffuse astrocytoma and

Received April 28, 2014; accepted after revision June 17.

From the Department of Cancer Imaging (A.L.), Peter MacCallum Cancer Centre, East Melbourne, Victoria, Australia; Departments of Pathology (A.T.), Neurosurgery (K.J.D.), Medical Oncology (K.M.F.), and Radiology (F.G.), The Royal Melbourne Hospital, Parkville, Victoria, Australia; and Melbourne EpiCentre (M.A.T.), Department of Medicine, The University of Melbourne and The Royal Melbourne Hospital, Parkville, Victoria, Australia.

Paper previously presented at: Annual Meeting of the Radiological Society of North America, December 1-6, 2013; Chicago, Illinois; and preliminary pilot data presented at: 14th Congress of the Asian Oceanian Congress of Radiology, August 30 to September 2, 2012; Sydney, Australia.

Please address correspondence to Arian Lasocki, MD, Department of Radiology, Peter MacCallum Cancer Centre, St Andrews Place, East Melbourne, Victoria, Australia 3002; e-mail: arian.lasocki@petermac.org

http://dx.doi.org/10.3174/ajnr.A4077 anaplastic astrocytoma, respectively) may also progress to grade IV (glioblastoma) with time. Secondary glioblastomas are associated with a better prognosis and typically demonstrate isocitrate dehydrogenase-1 (IDH1) mutations. ${ }^{2}$ Assigning a specific grade is important for routine patient management and for inclusion into clinical trials. The overall tumor grade depends on the highest grade component within the sample obtained; however, astrocytomas are heterogeneous tumors on both imaging and histology, and different components of the same tumor may exhibit different grades on histopathology. As a result, sampling error sometimes occurs when grading astrocytomas from surgical specimens, in particular when the specimen was obtained by biopsy. ${ }^{3-5}$ It also remains a risk with a resection because the pathologist typically does not receive the entire resected tumor for examination, as generally occurs with visceral tumors. The highest grade component may therefore not be present in the sample available for analysis. This sampling error can lead to undergrading of tumors, which, in turn, can potentially result in undertreatment. In 
addition, due to varying practices even among pathologists working within the same department, interobserver correlation for histopathology has been shown to be only poor to moderate $(\kappa=$ $0.06-0.66){ }^{6-8}$

MR imaging has been shown to correlate with histologic grade $^{9-14}$ but has the important advantage of being able to scan the entire tumor in vivo and is therefore not susceptible to sampling error. When evaluating MR imaging, the literature usually uses histologic grade as the criterion standard; this practice makes it difficult to interpret the results given the aforementioned limitations of histopathology (sampling error and inter- and intraobserver variability) and likely underestimates the accuracy and utility of MR imaging. Using histopathology as the criterion standard also does little to clarify the practical questions of how to proceed when histopathology and MR imaging are disparate and which of the 2 better predicts the patient's outcome in this situation. In particular, it is not clear whether a patient should be presumed to have a higher grade tumor when the MR imaging appearance suggests a higher grade than the histologic assessment.

In this study, we correlated MR imaging appearances with patient survival and compared how well the MR imaging grade predicted biologic behavior compared with the histologic grade. We proposed that incorporating MR imaging into grading could predict patient survival better than the current criterion standard of histopathology alone.

\section{MATERIALS AND METHODS Patient Selection}

Patients with a new diagnosis of a World Health Organization grade II-IV astrocytoma or mixed oligoastrocytoma were identified through the Central Nervous System Tumor Data Base at our hospital, which routinely captures all new patients with astrocytoma treated at our institution. All were adults who had not undergone treatment before surgery. Protoplasmic astrocytomas $(n=9)$ and gemistocytic astrocytomas $(n=5)$, which are known to have atypical imaging appearances, were excluded, as were patients with imaging appearances suggestive of gliomatosis cerebri $(n=4)$. Pure oligodendrogliomas were also not included. We included only patients with MR imaging performed before their first operation for this tumor, including at least a postcontrast sequence, available for review on the PACS. Seventeen patients were excluded due to a lack of preoperative postcontrast MR imaging. The study period was September 2007 to December 2010, to allow adequate time for survival information. Institutional $\mathrm{Hu}-$ man Research Ethics Committee approval was obtained.

Patient demographics and baseline Eastern Cooperative Oncology Group (ECOG) performance status were obtained from the data base. The histologic grade of the tumor was taken from the histopathology report available on the clinical information system of the hospital. One patient was excluded because the histopathology had been reported at another institution and was not available for review. Of the histology reports included, all except 1 patient's histology was reported by 1 of 2 experienced neuropathologists. Histology for this single outlying patient and for 6 patients for whom the histopathologic grade was not clearly stated in the hospital report were blindly reviewed and graded by 1 of the 2 neuropathologists for the purposes of the study. IDH1 mutation test- ing was subsequently performed for patients with evidence of necrosis on MR imaging but grade II or III histology, to determine if this subset of patients showed an association with IDH1 mutations, which would be suggestive of secondary glioblastomas. The proliferative index (Ki-67 or topoisomerase) was also determined for these patients, either taken from the formal hospital report $(n=10)$ or calculated on subsequent review if not reported initially $(n=13)$.

\section{MR Imaging Review}

Preoperative MRIs were independently reviewed retrospectively on the PACS by 2 readers, blinded to the histologic grade, and an MR imaging grade was given. The readers were an MR imaging fellow and a neuroradiologist with 5 years of subspecialty experience. For this study, the MR imaging grade was determined on the basis of the postcontrast MR imaging, as a surrogate for the histologic grading criteria used at our institution. MR imaging grade IV was assigned when there was evidence of necrosis-a complete enhancing ring on a postcontrast T1-weighted sequence around a central nonenhancing area. If there was enhancement but no convincing necrosis, this finding was designated grade III. Wispy enhancement was not considered sufficient for grade III. MR imaging grade II was assigned when the tumor demonstrated no contrast enhancement or necrosis. Discrepancies in the grade between the $2 \mathrm{MR}$ imaging readers were decided by consensus, with knowledge of their initial MR imaging grade but again blinded to the histopathologic grade.

Most MR imaging studies (236 patients) were performed entirely at our institution, on 1 of 3 scanners (Magnetom Tim Trio; Siemens, Erlangen, Germany, 3T, software VB 17; Signa HDx, 1.5T; GE Healthcare, Milwaukee, Wisconsin, software Version 14; Signa LX, 1.5T; GE Healthcare, software Version 9.1). Most (212 of 245) were standard diagnostic studies. At 1.5T, these generally included at least axial T1WI, T2WI, FLAIR, and DWI sequences; sagittal T1WI; and a susceptibility-sensitive sequence (varying among scanners). Postcontrast T1-weighted sequences were performed in the axial and coronal planes, and usually a volumetric acquisition for the purposes of stereotaxis was performed. At 3T, the only difference was that the pre- and postcontrast T1-weighted sequences were acquired volumetrically, with reconstructions in 3 planes. Overall, MR spectroscopy and dynamic susceptibility contrast perfusion were performed in 63 and 31 patients, respectively. The remainder $(n=33)$ were most commonly limited volumetric postcontrast T1WI studies for stereotaxis. The standard intravenous contrast medium administered was $15 \mathrm{~mL}$ of gadopentetate dimeglumine, with the postcontrast sequence generally performed 2-3 minutes after contrast administration.

\section{Surgical Technique}

Surgical biopsies and resections were guided stereotactically by targeting the most suspicious areas on MR imaging by using the VectorVision Navigation System (BrainLAB, Feldkirchen, Germany). This usually incorporated a volumetric postcontrast T1WI sequence. Information about whether the operation was a biopsy or resection was obtained from the data base.

\section{Statistical Analysis}

Interobserver reliability and agreement between the 2 readers of MR imaging grades were evaluated by using the $\kappa$ statistic, while 
the Spearman correlation coefficient was used to assess the correlation between the histology and MR imaging grades. Both univariate and multivariate Cox logistic regression analyses were used to test for associations between overall survival and age, sex, performance status, and a combination of MR imaging and histologic grades. ECOG performance status was considered in a binary manner, namely $0-1$ and $2-4$. This grouping was selected because it provided better balance between the 2 groups than the other of the 2 groupings commonly used in the literature: $0-2$ and 3-4. Different categoric variables were created that reflected different combinations of MR imaging and histologic grades. For the univariate analysis, the log-rank test was used for associations between survival and categoric variables, while Cox regression was used for continuous variables such as age. Kaplan-Meier curves were estimated to illustrate the association and nonassociations between survival and the categoric variables, with the Cox proportional hazard model used to compare survival between groups, after adjusting for relevant covariates. Results are presented as hazard ratios (HRs) with 95\% confidence intervals. A 2-tailed $P$ value of .05 was considered statistically significant. STATA statistical analysis software (Version 12.1; StataCorp, College Station, Texas) was used.

\section{RESULTS}

\section{Patient Demographics}

Two hundred forty-five patients met the inclusion criteria, of 281 patients identified initially. There were 136 men (56\%) and 109 women. Patient ages ranged from 19 to 89 years, with a median of 61 years. Patient age was found to be a strong predictor of survival independent of histologic grade; hence, all results provided are corrected for age. Forty patients (16\%) had grade II histology, 35 (14\%) had grade III, and 170 (69\%) had grade IV. Thirty-four patients (14\%) had mixed oligoastrocytomas; the remainder had astrocytomas $(n=211)$. All patients underwent MR imaging within 11 days before surgery, and all except 7 patients, within 7 days. ECOG performance status data were available in $91 \%$ of patients (223 of 245): Ninety-seven patients were ECOG 0; 60, ECOG 1; 33, ECOG 2; 25 , ECOG, 3; and 8, ECOG 4. As for age, performance status was an independent predictor of survival, thus the associations with survival for the MR imaging and histologic grades were also corrected for performance status. These baseline characteristics and the univariate results are presented on Table 1.

\section{MR Imaging Histology Correlation}

The MR imaging and histologic grades are compared in Table 2 for astrocytomas and in Table 3 for mixed oligoastrocytomas. Overall agreement between the $2 \mathrm{MR}$ imaging readers was high, occurring in 233 of 245 patients, or $95 \%(\kappa=0.87)$. Correlation between the MR imaging consensus grade and the histologic grade was moderate at $82 \%(\kappa=0.58)$.

\section{Astrocytomas}

Of the 25 patients with grade II histology, 5 (20\%) exhibited MR imaging evidence of necrosis (consistent with a grade IV tumor). Their mean survival was 233 days, significantly worse than the survival of patients with grade II histology but no evidence of necrosis on MR imaging (median survival, $>946$ days; $P=.002$;
Table 1: Patient baseline characteristics and univariate results

\begin{tabular}{|c|c|c|c|c|}
\hline Variable & $\begin{array}{c}\text { No. (\%), Unless } \\
\text { Otherwise } \\
\text { Specified }\end{array}$ & HR & $95 \% \mathrm{Cl}$ & $P$ Value \\
\hline Total & 245 & & & \\
\hline \multicolumn{5}{|l|}{ Sex } \\
\hline Female & $109(44.5 \%)$ & 1 & - & - \\
\hline Male & $136(55.5 \%)$ & 1.15 & $0.86-1.54$ & .346 \\
\hline \multicolumn{5}{|l|}{ Age (yr) } \\
\hline \multicolumn{5}{|l|}{ Continuous } \\
\hline Median (IQR) & $61(49-71)$ & 1.05 & $1.04-1.07$ & $<.001$ \\
\hline \multicolumn{5}{|l|}{ Categorized } \\
\hline $19-53$ & 79 (32.2\%) & 1 & - & - \\
\hline $54-67$ & $80(32.7 \%)$ & 2.59 & $1.75-3.84$ & $<.001$ \\
\hline $68+$ & $86(35.1 \%)$ & 5.05 & $3.42-7.47$ & $<.001$ \\
\hline \multicolumn{5}{|l|}{ MRI grade } \\
\hline II & $39(15.9 \%)$ & 1 & - & - \\
\hline III & $19(7.8 \%)$ & 2.28 & $0.97-5.38$ & .059 \\
\hline IV & $187(76.3 \%)$ & 8.39 & $4.50-15.67$ & $<.001$ \\
\hline \multicolumn{5}{|l|}{ Histology grade } \\
\hline ॥ & $40(16.3 \%)$ & 1 & - & - \\
\hline III & $35(14.3 \%)$ & 2.55 & $1.28-5.08$ & .007 \\
\hline IV & $170(69.4 \%)$ & 5.88 & $3.32-10.43$ & $<.001$ \\
\hline \multicolumn{5}{|l|}{ Histology type } \\
\hline Astrocytoma & 211 (86.1\%) & 1 & - & - \\
\hline Oligoastrocytoma & 34 (13.9\%) & 0.31 & $0.18-0.52$ & $<.001$ \\
\hline \multicolumn{5}{|l|}{ ECOG (continuous) } \\
\hline Mean \pm SD & $0.78 \pm 1.39$ & 1.47 & $1.30-1.67$ & $<.001$ \\
\hline \multicolumn{5}{|l|}{ ECOG (grouped) } \\
\hline 1 & $157(64.1 \%)$ & 1 & - & - \\
\hline $2-4$ & $66(26.9 \%)$ & 2.13 & $1.54-2.94$ & $<.001$ \\
\hline
\end{tabular}

Note:- IQR indicates interquartile range.

Table 2: Number of patients with astrocytoma with each histologic and MRI grade

\begin{tabular}{lrrrr}
\hline \multirow{2}{*}{$\begin{array}{l}\text { MRI } \\
\text { Grade }\end{array}$} & \multicolumn{3}{c}{ Histologic Grade } & \\
\cline { 2 - 4 } II & 15 & 5 & III & Total \\
\hline III & 5 & 7 & 4 & 21 \\
IV & 5 & 16 & 153 & 16 \\
Total & 25 & 28 & 158 & 174 \\
\hline
\end{tabular}

Table 3: Number of patients with mixed oligoastrocytoma with each histologic and MRI grade

\begin{tabular}{lrcrc}
\hline \multirow{2}{*}{ MRI } & \multicolumn{3}{c}{ Histologic Grade } & \\
\cline { 2 - 4 } Grade & II & III & IV & Total \\
\hline II & 13 & 5 & 0 & 18 \\
III & 1 & 1 & 1 & 3 \\
IV & 1 & 1 & 11 & 13 \\
Total & 15 & 7 & 12 & 34 \\
\hline
\end{tabular}

$\mathrm{HR}=44.6$; 95\% CI, 4.0-502.6). These patients with necrosis on MR imaging had statistically equivalent survival $(P=.164)$ compared with those with grade IV histology (median survival, 257 days), though with a slight trend toward worse survival (HR = $1.9 ; 95 \% \mathrm{CI}, 0.8-4.8$ ). The survival of these 3 groups is compared on the Kaplan-Meier curve (Fig 1).

For the grade III histology group, 16 of 28 (59\%) had evidence of necrosis on MR imaging, with a median survival of 245 days. Examples are shown in Fig 2. Again, their survival was significantly worse than that for patients without MR imaging evidence of necrosis (median survival, 564 days; $P=.029 ; \mathrm{HR}=3.3 ; 95 \%$ CI, 1.1-9.8) and was equivalent to that of the grade IV histology 
group $(P=.385)$. These survivals are compared on the KaplanMeier curve (Fig 3).

IDH1 mutation status was tested on all 21 patients with grade II or III histology but MR imaging evidence of necrosis, and it was negative in all patients. The proliferative index varied widely, from $1 \%$ to $>75 \%$. Five of the 21 patients had further surgery within 4 months of their initial surgery (after 8, 10, 46, 90, and 109 days, respectively). In each case, histology showed grade IV tumor. Histology was obtained by resection in 9 of 21 patients (43\%) with grade II or III histology but MR imaging evidence of necrosis and by biopsy in the other 12 . This proportion obtained by resection was slightly higher than that for the patients with grade II or III histology but no evidence of necrosis on MR imaging $(11$ of $32,34 \%)$. This difference was not statistically significant $(P=.533)$.

Of patients with grade II histology, there was no significant difference in survival $(P=.300)$ between those with no enhancement (MR imaging grade II) compared with those with enhancement but no evidence of necrosis (MR imaging grade III). Similarly, for patients with grade III histology, there was no significant difference in survival $(P=.119)$ between those with MR imaging grades of II and III. Of the patients with grade IV histology, imaging of 4 demonstrated enhancement but not convincing necrosis on MR imaging, and imaging of 1 patient did not exhibit any enhancement. These 5 patients without evidence of necrosis on

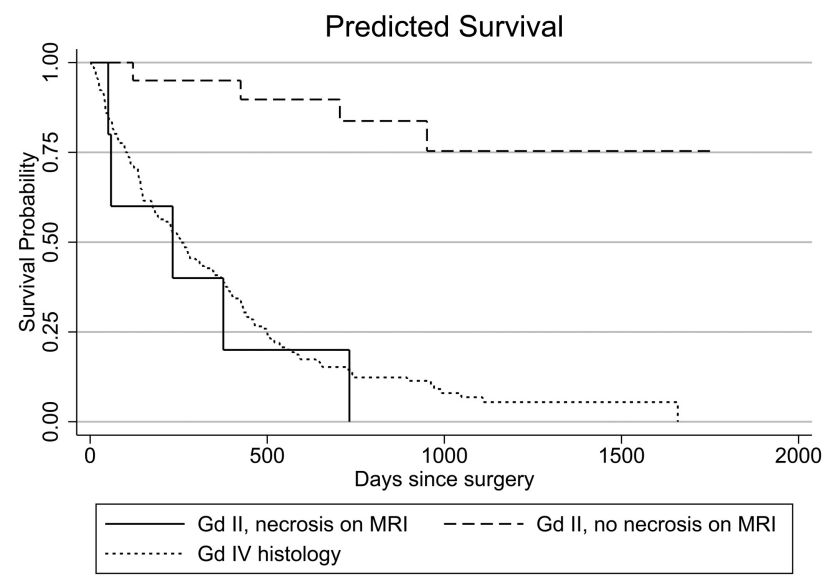

FIG 1. Kaplan-Meier survival curve showing survival of patients with grade II astrocytomas on histology, with and without evidence of necrosis on MR imaging, compared with patients with grade IV histology.

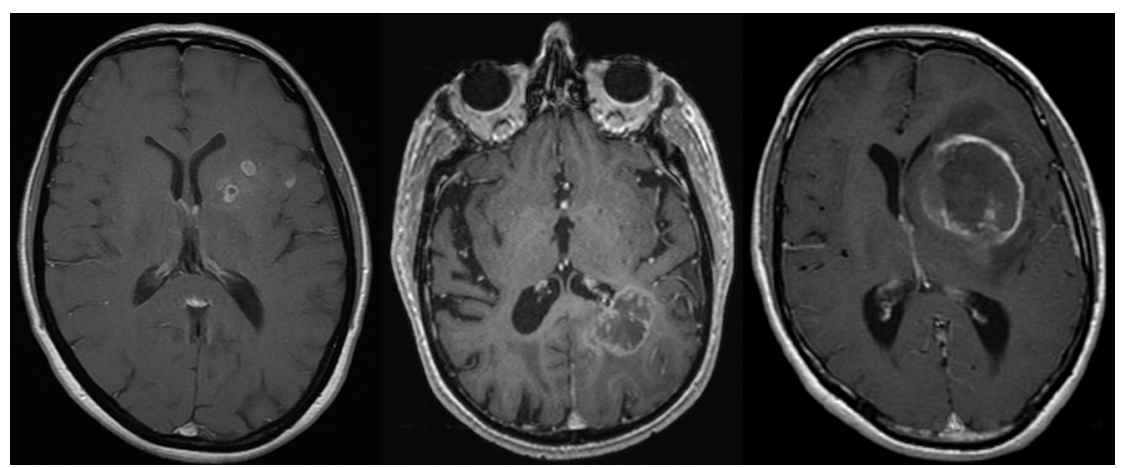

FIG 2. Examples of postcontrast TI-weighted sequences of patients with grade III astrocytomas on histology but MR imaging evidence of necrosis.
MR imaging showed a trend toward longer survival (median survival, 589 days) compared with the remaining patients with grade IV histology (median survival, 252 days; $\mathrm{HR}=0.4 ; 95 \%$ CI, $0.1-$ $1.3)$, though with the small number of patients, this was not statistically significant $(P=.138)$.

\section{Mixed Oligoastrocytomas}

Fewer grade II and III oligoastrocytomas showed evidence of necrosis on MR imaging (only 1 of each grade); this result precluded adequate subgroup analysis. IDH1 was positive in 1 of these 2 patients. When corrected for age, ECOG performance status, and histologic grade, patients with mixed oligoastrocytomas had significantly better survival than those with pure astrocytomas $(P=$ $.031 ; \mathrm{HR}=0.52 ; 95 \% \mathrm{CI}, 0.3-0.9)$.

\section{DISCUSSION}

We have described a simple yet robust approach to MR imaging grading of intracranial astrocytomas. MR imaging can accurately identify a significant number of patients undergraded by histology, in particular those suspected of truly having grade IV tumors on the basis of MR imaging evidence of necrosis. The survival data strongly suggest that all or most of the astrocytomas with grade II and III histology but evidence of necrosis on MR imaging were indeed glioblastomas. In addition, they appear to have generally been primary glioblastomas, given IDH1 mutation negativity, rather than secondary glioblastomas (which are associated with IDH1 mutations and typically have a better prognosis). ${ }^{2}$ Undergrading occurred with both stereotactic biopsies and definitive resections. We have shown that MR imaging has excellent interobserver agreement, better than the inter- and intraobserver agreement reported for pathologic grading of astrocytomas $(\kappa=$ $0.06-0.66) .{ }^{6-8}$ In addition, combining MR imaging studies from different scanners indicates that these results using standard sequences should be achievable in most institutions, rather than being vendor- or model-specific. Finally, the high correlation between a relatively inexperienced reader and a more experienced reader shows that the grading system can be easily used by radiologists of varying experience.

We consider grade IV the most important histologic grade to determine accurately at diagnosis because the management is usually more aggressive than for grade III and, in particular, grade II tumors, ${ }^{15-17}$ and patients can deteriorate rapidly. Correct identification of grade IV tumors allows appropriate intensification of treatment to a multimodality regimen at diagnosis. Patients with grade IV tumors are also those who are potential candidates for the greatest number of clinical trials and novel therapies. Clinical trial participation has been shown to be an independent predictor of longer survival in glioblastoma ${ }^{18}$; hence, being ineligible for a trial due to undergrading has the potential to adversely affect a patient's outcome.

Our results build on work by authors at least as far back as Dean et al, ${ }^{9}$ who, in 
1990, aimed to grade astrocytic tumors on the basis of a variety of MR imaging features. Already $>2$ decades ago, they postulated that "MR imaging may serve as an adjunct in case management when the clinical course and MR findings appear to be at odds with the neuropathologic diagnosis." ${ }^{\prime 9}$ Our results are similar to a much more recent study of 151 patients by Pope et al, ${ }^{19}$ in which patients with grade III histology but MR imaging evidence of necrosis ( 8 of 43 grade III tumors, 19\%) had survival similar to that in patients with grade IV tumors, and 105 of 110 histologically proved grade IV tumors had MR imaging evidence of necrosis. In our study, the number of patients with grade III histology but MR imaging evidence of necrosis was greater ( 16 of $28,57 \%$ ). We have also shown a similar difference in survival for patients with histologic grade II tumors and evidence of necrosis on MR imaging (5 of $25,20 \%$ ), which was not examined by Pope et al.

Previous articles have shown overall moderate correlation between an MR imaging grade by using conventional sequences and the histopathologic grade, ${ }^{9-14,20}$ which improves when adding advanced imaging sequences such as perfusion (in particular relative cerebral blood volume) or spectroscopy. ${ }^{10,11,13,14,21-24}$ The results for DWI have been conflicting, ${ }^{13,21}$ as they have for the presence

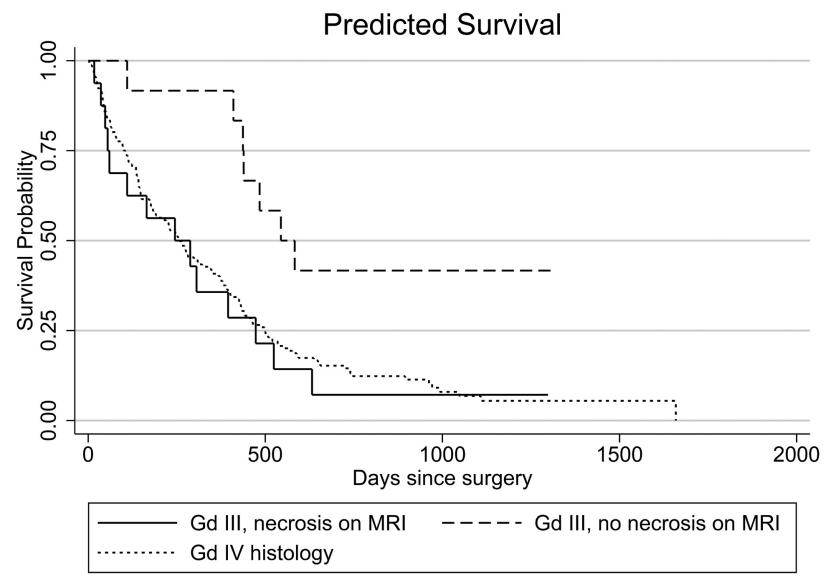

FIG 3. Kaplan-Meier survival curve showing survival of patients with grade III astrocytomas on histology, with and without evidence of necrosis on MR imaging, compared with patients with grade IV histology.

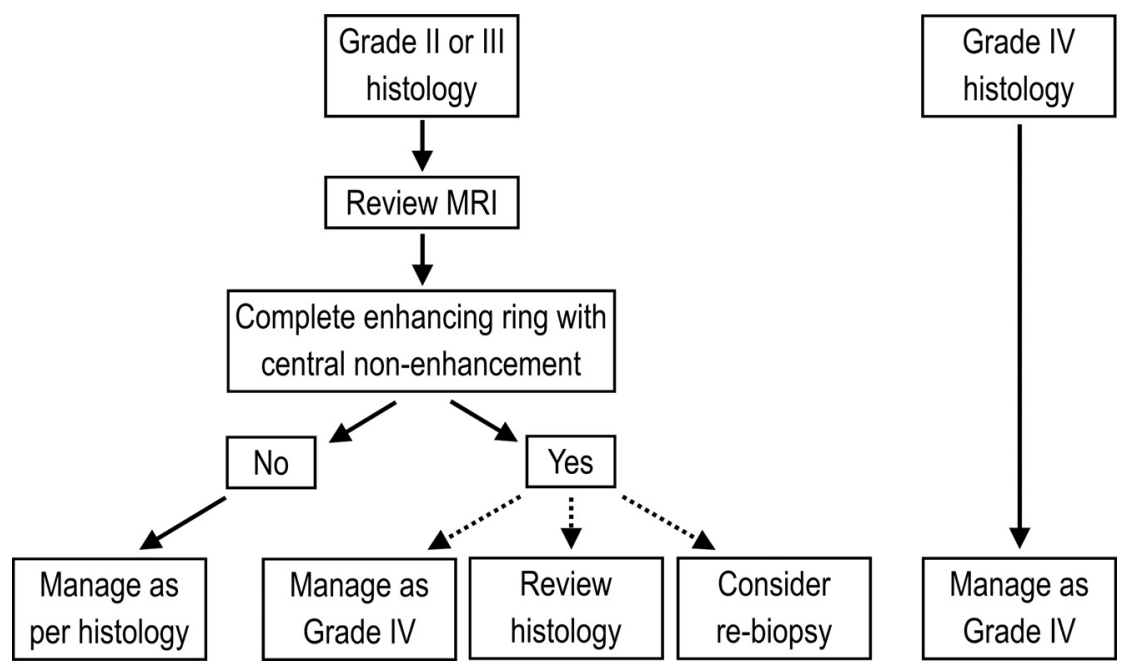

FIG 4. Suggested management algorithm for a combined histologic/MR imaging grading system. of magnetic susceptibility suggestive of hemorrhage. ${ }^{25,26} \mathrm{~A}$ practical difficulty with the advanced sequences is that they are generally continuous variables, with at least some overlap between tumors of different grades. It is likely that the degree of overlap between tumor grades would substantially increase once those tumors with MR imaging evidence of necrosis are excluded; this change would decrease the specificity and thus the utility of these sequences. A further difficulty with many of the studies of MR imaging grading of astrocytic tumors is that grade III and IV tumors have been considered together as "high-grade gliomas." $11,13,14,21-23,25,27,28$ In general, distinction between grade III and IV tumors was inaccurate ${ }^{11,27}$ or was not assessed, yet this distinction can be very important for determining both management options and clinical trial inclusion. Not unexpectedly, our simple grading system was less helpful in differentiating grade II and III tumors on the basis of the presence or absence of enhancement, and this is likely to be a setting in which the advanced sequences will have particular value.

We acknowledge that management may have varied substantially among patients. Age and performance status are both important factors in the decision-making process, and we think that correcting for these variables addresses this variation in management between patients, especially given that ours is a single-institution study in the post-Stupp era. ${ }^{17}$ During the study period, our postsurgery standard therapy for patients with grade IV tumors was combination temozolomide and radiation therapy followed by adjuvant temozolomide; patients with grade III tumors were initially treated with surgery and postoperative radiation therapy, followed by the introduction of temozolomide when there was evidence of recurrence, while patients with grade II tumors were generally managed with surgery alone, followed by active surveillance until evidence of dedifferentiation was seen. Molecular and genetic markers such as IDH1 mutation status, proliferative index, and O6-methylguanine DNA methyltransferase methylation were not typically obtained during the study period and were not used to guide treatment. A further potential criticism of this study is that advanced imaging sequences were underused in determining the MR imaging grade. Although advanced sequences can be beneficial in grading, our aim was to develop a fairly direct MR imaging correlate for the WHO classification, which was simple and reproducible by all institutions. Additionally, given that we have shown that the presence of necrosis on MR imaging (as a surrogate for necrosis on histopathology) is sufficient to identify most grade IV tumors, including those suspected of being undergraded due to sampling error, it is not clear from the literature how useful the advanced sequences are for assessing tumors that do not have evidence of necrosis on the postcontrast T1-weighted sequence.

Our patient group consisted of those with a higher proportion of grade IV tumors than some other studies. This may partly relate to our exclusion of patients with protoplasmic and gemistocytic 
variants and those suspected on MR imaging of having gliomatosis cerebri. It is not clear whether these patients were included in other studies. We considered it important to exclude these histologic variants from our study because they have imaging appearances that vary significantly from the more common diffuse fibrillary variety (contrast enhancement and cystic change being far more common), and in the case of gemistocytic astrocytomas, the prognosis is worse. ${ }^{1,29,30}$ The patients suspected of having gliomatosis cerebri were also excluded for this same reason of having a worse prognosis because including them could have unfairly biased against histology. Nevertheless, due to the size of our study, the absolute number of grade II and III tumors was large compared with that in the existing literature.

The management of astrocytomas has progressed beyond just histologic assessment, with molecular and genetic markers being able to provide information on prognosis and expected treatment outcomes. ${ }^{31}$ The interpretation and implications of the genetic analysis are affected by the histologic grade, however; hence, it remains important to optimize the accuracy of grading. Accurate grading becomes even more important when these markers are not available (for example, due to cost). In either case, using MR imaging to improve the accuracy of grading can help optimize management and prognostication with little or no added cost because an MR imaging has generally already been performed.

The algorithm we suggest for incorporating an MR imaging grade into the overall management (Fig 4) hinges on all patients with grade II and grade III astrocytoma having their preoperative MR imaging reviewed for grading, with the radiologist aware of the histology but potentially blinded to the histologic grade. If the MR imaging does show evidence of necrosis, our results suggest that the tumor can simply be treated as grade IV, with the appropriate multimodality therapy. If histologic confirmation of grade IV tumor is necessary, we suggest reviewing the histology in the first instance, specifically looking for evidence of necrosis to verify the MR imaging appearances. If no necrosis is revealed on review, repeat surgery could also be considered, though in practice, it would be a last resort. The results for astrocytomas were not reproduced for oligoastrocytomas due to the smaller numbers and less frequent upgrading of tumors based on MR imaging.

\section{CONCLUSIONS}

Using MR imaging in tandem with conventional histologic grading can significantly enhance the accuracy of grading of intracranial astrocytomas, with high interobserver agreement. Detection of necrosis on MR imaging in a histologic grade II or III astrocytoma suggests that the patient has been undergraded by histology. This result has the potential to optimize patient management, for example, by encouraging a more aggressive treatment strategy earlier in the patient's course.

Disclosures: Arian Lasocki—RELATED: Support for Travel to Meetings for the Study or Other Purposes: Radiology Resources (prize awarded by the Royal Australian and New Zealand College of Radiologists). Kathryn M. Field_UUNRLATED: Payment for Lectures (including service on Speakers Bureaus): Roche; Travel/Accommodations/ Meeting Expenses Unrelated to Activities Listed: Merck Sharp and Dohme. Frank Gaillard-UNRELATED: Consultancy: Medtronic, Comments: educational talks about MRI in the context of MRI-safe pacemakers and neurostimulators; Employment: Radiopaedia.org, Comments: Founder, Editor, CEO of Radiopaedia.org, an online collaborative radiology resource, www.radiopaedia.org.

\section{REFERENCES}

1. Louis DN. ed. WHO Classification of Tumours of the Central Nervous System. 4th ed. Lyon: International Agency for Research on Cancer; 2007

2. Ohgaki $\mathrm{H}$, Kleihues $\mathrm{P}$. The definition of primary and secondary glioblastoma. Clinical Cancer Research 2013;19:764-72

3. Chandrasoma PT, Smith MM, Apuzzo ML. Stereotactic biopsy in the diagnosis of brain masses: comparison of results of biopsy and resected surgical specimen. Neurosurgery 1989;24:160-65

4. Glantz MJ, Burger PC, Herndon JE, et al. Influence of the type of surgery on the histologic diagnosis in patients with anaplastic gliomas. Neurology 1991;41:1741-44

5. Jackson RJ, Fuller GN, Abi-Said D, et al. Limitations of stereotactic biopsy in the initial management of gliomas. Neuro Oncol 2001; 3:193-200

6. Coons SW, Johnson PC, Scheithauer BW, et al. Improving diagnostic accuracy and interobserver concordance in the classification and grading of primary gliomas. Cancer 1997;79:1381-93

7. Mittler MA, Walters BC, Stopa EG. Observer reliability in histological grading of astrocytoma stereotactic biopsies. J Neurosurg 1996;85:1091-94

8. Prayson RA, Agamanolis DP, Cohen ML, et al. Interobserver reproducibility among neuropathologists and surgical pathologists in fibrillary astrocytoma grading. J Neurol Sci 2000;175:33-39

9. Dean BL, Drayer BP, Bird CR, et al. Gliomas: classification with MR imaging. Radiology 1990;174:411-15

10. Knopp EA, Cha S, Johnson $G$, et al. Glial neoplasms: dynamic contrast-enhanced $\mathrm{T} 2{ }^{*}$-weighted $\mathrm{MR}$ imaging. Radiology 1999;211:791-98

11. Law M, Yang S, Wang H, et al. Glioma grading: sensitivity, specificity, and predictive values of perfusion MR imaging and proton MR spectroscopic imaging compared with conventional MR imaging. AJNR Am J Neuroradiol 2003;24:1989-98

12. Möller-Hartmann W, Herminghaus S, Krings T, et al. Clinical application of proton magnetic resonance spectroscopy in the diagnosis of intracranial mass lesions. Neuroradiology 2002;44:371-81

13. Arvinda HR, Kesavadas C, Sarma PS, et al. Glioma grading: sensitivity, specificity, positive and negative predictive values of diffusion and perfusion imaging. J Neurooncol 2009;94:87-96

14. Kim HS, Kim SY. A prospective study on the added value of pulsed arterial spin-labeling and apparent diffusion coefficients in the grading of gliomas. AJNR Am J Neuroradiol 2007;28:1693-99

15. Brown PD. Low-grade gliomas: the debate continues. Curr Oncol Rep 2006;8:71-77

16. DeAngelis LM. Anaplastic glioma: how to prognosticate outcome and choose a treatment strategy. J Clin Oncol 2009;27:5861-62

17. Stupp R, Mason WP, van den Bent MJ, et al. Radiotherapy plus concomitant and adjuvant temozolomide for glioblastoma. $N \mathrm{Engl}$ J Med 2005;352:987-96

18. Field KM, Drummond KJ, Yilmaz M, et al. Clinical trial participation and outcome for patients with glioblastoma: multivariate analysis from a comprehensive dataset. $J$ Clin Neurosci 2013;20:783-89

19. Pope WB, Sayre J, Perlina A, et al. MR imaging correlates of survival in patients with high-grade gliomas. AJNR Am J Neuroradiol 2005;26:2466-74

20. Chishty IA, Rafique MZ, Hussain M, et al. MRI characterization and histopathological correlation of primary intra-axial brain glioma. Journal of the Liaquat University of Medical and Health Sciences 2010;9:64-69

21. Zonari P, Baraldi P, Crisi G. Multimodal MRI in the characterization of glial neoplasms: the combined role of single-voxel MR spectroscopy, diffusion imaging and echo-planar perfusion imaging. $\mathrm{Neu}$ roradiology 2007;49:795-803

22. Chawla S, Wang S, Wolf RL, et al. Arterial spin-labeling and MR spectroscopy in the differentiation of gliomas. AJNR Am J Neuroradiol 2007;28:1683-89

23. Bisdas S, Kirkpatrick M, Giglio P, et al. Cerebral blood volume mea- 
surements by perfusion-weighted MR imaging in gliomas: ready for prime time in predicting short-term outcome and recurrent disease? AJNR Am J Neuroradiol 2009;30:681-88

24. Meyerand ME, Pipas JM, Mamourian A, et al. Classification of biopsy-confirmed brain tumors using single-voxel MR spectroscopy. AJNR Am J Neuroradiol 1999;20:117-23

25. Pinker K, Noebauer-Huhmann IM, Stavrou I, et al. High-resolution contrast-enhanced, susceptibility-weighted $\mathrm{MR}$ imaging at $3 \mathrm{~T}$ in patients with brain tumors: correlation with positron-emission tomography and histopathologic findings. AJNR Am J Neuroradiol 2007;28:1280-86

26. Asari S, Makabe T, Katayama S, et al. Assessment of the pathological grade of astrocytic gliomas using an MRI score. Neuroradiology 1994;36:308-10

27. Bulakbasi N, Kocaoglu M, Örs F, et al. Combination of single-voxel proton MR spectroscopy and apparent diffusion coefficient calculation in the evaluation of common brain tumors. AJNR Am J Neuroradiol 2003;24:225-33

28. Bulakbasi N, Kocaoglu M, Farzaliyev A, et al. Assessment of diagnostic accuracy of perfusion MR imaging in primary and metastatic solitary malignant brain tumors. AJNR Am J Neuroradiol 2005; 26:2187-99

29. Krouwer HG, Davis RL, Silver P, et al. Gemistocytic astrocytomas: a reappraisal. J Neurosurg 1991;74:399-406

30. Avninder S, Sharma M, Deb P, et al. Gemistocytic astrocytomas: histomorphology, proliferative potential and genetic alterations: a study of 32 cases. J Neurooncology 2006;78:123-27

31. Thomas La, Di Stefano AL, Ducray F. Predictive biomarkers in adult gliomas: the present and the future. Curr Opin Oncol 2013;25: $689-94$ 